\title{
Evaluation of Emerging Audio Description Systems for Broadcast TV
}

\author{
Anna Vilaro, Aitor Rodriguez-Alsina, Pilar Orero, and Jordi Carrabina \\ CAIAC, Universitat Autònoma de Barcelona, Spain. \\ \{anna.vilaro, aitor.rodriguez, pilar.orero, \\ jordi.carrabina\} @uab.cat
}

\begin{abstract}
Following EU directives on Media Access and Ambient Assisted Living, the broadcasting industry needs to introduce new services in order to guarantee access to all citizens. The article and its conclusions are part of the EU project DTV4ALL, which focuses on some possible broadcasting scenarios for achieving barrier-free television for those with visual impairments. Five enhanced Audiodescription (AD) scenarios were proposed and evaluated: 1) Live streaming Internet TV with AD, 2) AD reception in a group situation, 3) Video on demand over a set-top-box, 4) Video on PC and 5) Podcasts. User evaluation concerning usefulness, quality and usability of the services was assessed using questionnaires. Results of the user evaluation show that not only are AD emerging services technically viable but they are also positively rated by users. Implementation of these services will provide improved access to content, making TV accessible for all.
\end{abstract}

Keywords: accessibility, audio description, emerging services, user evaluation, ambient assisted living, elderly.

\section{Introduction}

Ambient Assisted Living (AAL) defines the solutions for the enhancement of the quality of life of older people based on the information and communication technologies. The home environment is a significant space for the family conciliation and, while the technology of the consumer electronics for the home environment is evolving rapidly, the risk of exclusion of elderly people increases. Some of this barriers concern to the accessibility of multimedia content and, in particular, to audiovisual content. In this context, technology must be especially designed taking into account the user needs rather than in the technology itself [1].

People who are blind or partially sighted need audio description (AD) to be provided with a television program if they are to fully appreciate the context of what they hear. Nowadays, an AD track is broadcasted within the TV stream as a separated audio track [2] [3] that users can select with the remote control. AD track contains the 
original audio content mixed with a voice-over track giving further explanation about what is happening on the screen [4]. This service is provided in some countries such as the UK, where the rate of $\mathrm{AD}$ has been raised to $20 \%$ in recent years. This rate varies across Europe [5], with some countries not offering any AD, and some beginning to raise their content to $10 \%$ of total output - the share recommended by the Spanish Government [6]. This is the case for Television of Catalonia (TVC).

TV broadcasters have also been deploying their content on different distribution networks such as the Internet or IPTV in order to enhance user engagement and user benefit from the new features they can offer. Since IPTV promotes a user-centric model of content broadcasting, it should provide suitable and improved accessibility services to ensure universal access for people who are deaf or visually impaired [7] as well as elderly people [8]. However, AD for video content seems to be less widespread in IP networks compared to broadcast systems, due to technical limitations of some distribution formats. These limitations require a higher implementation effort that has to be assumed by each content producer. In order to facilitate the provision of access services on TV, the European Union funded the project Digital Television for All (DTV4All) where TVC along with UAB undertook this study.

- The first objective is to identify significant emerging AD access services to cover all possible situations in a domestic environment. These services have to address the needs of disabled people and deliver services that are not yet on the market but are expected to become available in the near future. In this article we introduce five emerging AD scenarios for receiving AD. The scenarios simulated different audiovisual consumption situations and were aimed at evaluate the services.

- The second objective is to evaluate the users' perceived value of the proposed services. Specially, how attractive the new service is (its Usefulness), how easy it is to interact with the system (its Usability), and the general quality of the content received (its Quality). The results presented here are the first stage in the validation process of the services. We wanted to assess as many aspects of the services as possible. In particular, the quality of the broadcasted image and audio. This approach requires ensuring that users did not present any visual or hearing impairment. For this reason, people with visual or hearing impairments were not included in the participants' group in this stage. Taking into account that the target users of AD services are visually impaired people, a second stage of user evaluation will be carried out in the future involving visually impaired participants and an improved version of the services in order to evaluate specific aspects concerning its use by visually impaired users.

\section{Evaluation Methodology}

Fourteen participants were recruited as volunteers. Audio described content was provided by TVC. TV series named "La Riera", which is broadcasted using DTT signal, was used as the reference content in terms of quality of service. 
Five questionnaires were specially designed to evaluate each scenario. Three features concerning the scenarios were evaluated: usefulness, usability, and quality. The usability scale was adapted from Lewis questionnaire "post-study system usability questionnaire" [10]. Different types of questions were used: (1) Likert scale questions consisting of seven alternative responses ranging from "strongly disagree" to "completely agree". A normalization procedure was applied in the results in order to present the ratings in a 0-10 scale, (2) Comparative questions were presented in order to rank the features of the new system against those of the current system, and (3) Openanswer questions concerning the pros and cons of the system, as well as space for comments and suggestions.

Information about the tests and the objectives of the session were provided in written mode. Users were asked to carry out a specific task for each scenario and individually answer the corresponding questionnaire. A training phase was conducted to familiarize participants with the equipment and the assessment procedure.

\section{Evaluation}

According to the project plan, TVC delivered 5 prototypes of AD emerging services. The evaluated service descriptions and the user evaluation results are detailed below.

\subsection{Service description and results}

- Service 1: Live Streaming of Internet TV

This service emulates Digital Terrestrial Television (DTT) broadcasting by means of Internet (figure 1). Audio described content is transmitted in live mode via streaming from media servers located at TVC facilities. The signal emitted includes a high quality video signal and a minimum of two audio signals: a stereo Catalan audio channel and a mono AD channel, which is a mix of the normal audio and the AD created by the broadcaster. The content is encoded and packaged at the origin with the same quality as (DTT). The end-user will need a customized configured set-top-box to receive and decode the content instead of the current DTT air signal.

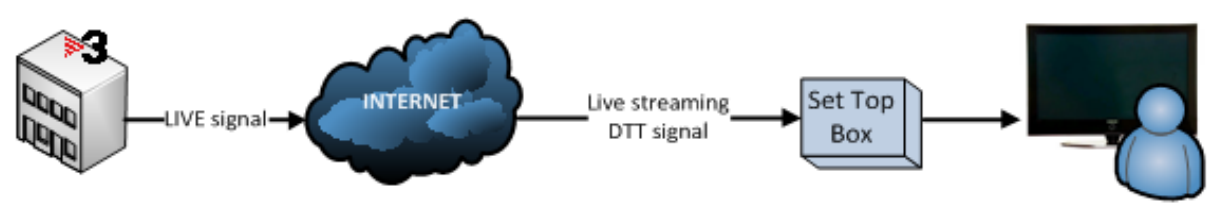

Fig. 1. Live Streaming of Internet TV

Participants watched the TV program 2-3 minutes using DTT signal first and using Internet TV afterwards. Their task was to activate the AD track with the remote control. 
Results show that, in general, the system was rated by participants as being "very useful". The rating included functionality and satisfaction attained. For example, the statement "In general, I think the service is useful" obtained a mean value of 8.8 points out of 10. Similarly, "The system has all the features and capabilities I expect" and "In general, I am satisfied with the service" both received a mean rating of 8.1. Quality was assessed by means of four Likert scale questions concerning image and audio quality as well as velocity to access the service. It was positively evaluated by participants. For example, users were very satisfied with the speed with which they could access the service (mean rating of 8.5). However, there are aspects of the service that need to be improved regarding Internet connection. Providing a constant and sufficient Internet bandwidth will guarantee good image quality. The global mean value for usability was 9.1. Users highlighted the ease of use and the quick access to the service as well as the cleanness of the graphic interface. Regarding the openanswer questions, users mentioned the system's usefulness for people with visual disabilities. They also pointed out "clarity of command" and that it "is friendly and easy to use". This latter aspect was highlighted by the majority of the users. Some suggestions were made regarding the incorporation of information about the content provided. For example, the inclusion of an audio described synopsis of the films will make the service more accessible.

- Service 2: AD reception in a group situation

This service proposes a solution for consuming television in group or family situations when one member of the group suffers from a visual impairment and requires AD. Figure 2 depicts the proposed service.

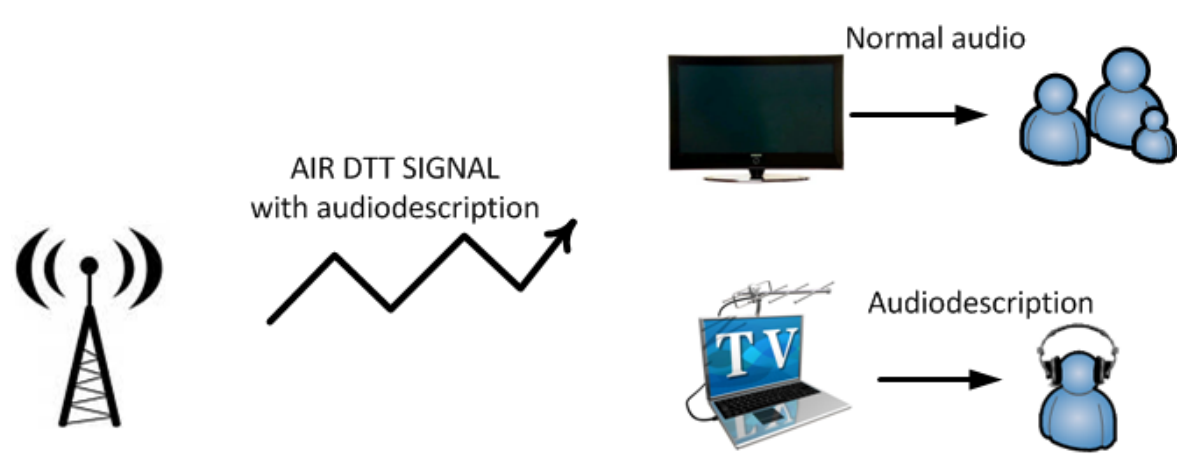

Fig. 2. AD Reception in a Group Situation.

The proposed service allows different users to watch the same TV screen together while the disabled user receives the AD channel individually through headphones connected to a laptop with a DTT tuner. It deals with simultaneous reception of media content both with and without AD. We wanted to test feasibility of the service and ensure that it does not involve problems with audio synchronization. The user received the AD through headphones connected to a laptop while other people received the standard audio channel. As this scenario focuses on the audio reception, only use- 
fulness and quality were evaluated. Participants were told to answer having in mind that the question referred to the situation where a visually impaired person is watching the audiovisual contents together with the participant.

Usefulness questions were presented as follows: 1) In general, I think this service is useful; 2) I would like to have this service; 3) If I had the service, I would use it; and 4) In general, I am satisfied with the system. In addition, participants were asked three open-answer questions regarding pros, cons and suggested improvements. The system was evaluated as very useful (gaining a mean rating of 8.6 in question 1) since it allows visually impaired people to have AD individually while the rest of the family consumes the content without. Users also pointed out that the system presents an exciting solution for families and the personalization of content. To evaluate the quality of image and sound, users answered a question concerning the overall quality rating in terms of: 1) bad; 2) mediocre; 3) satisfactory; 4) good or 5) excellent. They also answered five Likert scale questions about quality: 1) The audio quality of my headphones is good; 2) The sound does not experience cuts or breaks; 3) The audio from my headphones and the audio from the TV are presented at the same time; 4) No mismatches occur between the headphones and the TV; and 5) After selecting the service, access to content is quick. The overall quality was rated as "Good" by most users $(64 \%)$, with $22 \%$ considering it "Excellent". The mean rating for the Likert scale questions was 8.9. The positive evaluation validates this service as a possible solution for family conciliation in cases where a person suffers a visual impairment. Its acceptance has been demonstrated both in the technical aspect and in its application. However, some suggestions were made regarding the size of the laptop and the cable logistics that can be easily solved by using smaller devices and wireless connections.

- Service 3: Video on Demand to a Set-Top-Box.

The service permits to choose a recorded program from a list (i.e. 'catch-up') at any time. All the files will be saved in a repository and will be available to be served whenever users ask for them. The hybrid set-top-box used in the test allows the user to access the website to choose the video content he or she wishes to watch, as well as the audio he or she wants to listen to: either a standard Catalan or AD channel. Content is broadcasted via the Internet through a streaming IP channel. Task was to select a program from the catch-up menu and evaluate the system, focusing on the ease of achieving the desired content as well as evaluating the perceived quality of the video. The following diagram (figure 3 ) shows the distribution channel:

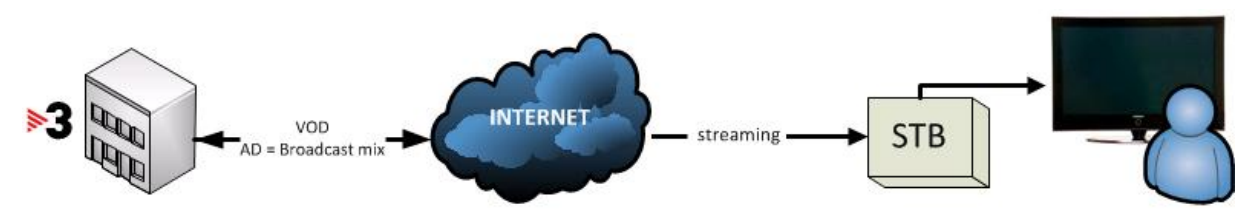

Fig. 3. Video On demand via set-top-box. 
A questionnaire with five Likert scale questions and two open-answer questions regarding pros and cons was administered. Service usefulness was rated as 8.9 out of 10. Users also highlighted the fact that access is instantaneous (streaming), so there is no waiting time. For example, one user mentioned as useful the fact that you can "access at any time to your favorite program without waiting" or "do not need to download and save the chapter". All these features make video on demand to a set-top-box a very attractive solution for $\mathrm{AD}$ reception. Figure 4 shows the selection menu screens for Video on demand system. Image and sound quality were evaluated through a question with five alternative answers. Most users declared that the quality was "Good" (64\%), whereas 29\% declared that it was "Satisfactory" and $7 \%$ said it was "Excellent". The usability assessment included questions concerning "ease of use" and "graphic interface". Data from the questionnaire showed that both aspects of usability obtained a good rating. The global mean rating for system usability was 9.2. Regarding the pros of the system, users emphasized the ease and speed of access to the video on demand service with AD. They also highlighted the temporal flexibility provided by the availability of chapters at any time and the possibility of watching a program more than once. Furthermore, some suggestions were made for improving the service. For example, users pointed out that there is no access to a time bar to advance or rewind the content.

- Service 4: Downloading videos on the PC.

This service allows the user to download media files on a PC so that these can be played on the same PC or on another multimedia device. The user will be able to download the content by accessing a web page with links to the AD programs. Participants' task was to browse the menu and select a program to download. The content was played on the computer using the VLC playback software [11]. The following schema (figure 4) shows the distribution channel.

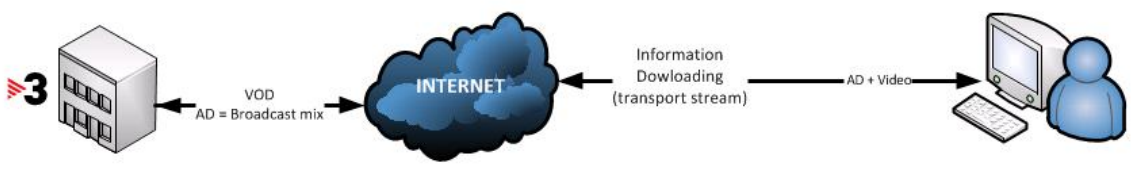

Fig. 4. Downloading videos on the PC

Users rated the general usefulness as 9.1 out of 10 . The possibility to play AD content on mobile devices was also appreciated by users. General quality was rated as "satisfactory" by $50 \%$ of users, as "good" by $36 \%$, and as "excellent" by $14 \%$. Image quality was rated as 8.4. Users emphasized the fact that the service guarantees excellent picture and sound quality as the service is provided directly by the broadcasters. However the time needed to download the files obtained the lowest value (6.7). The implementation of a more efficient download manager that allows the user to manage multiple files simultaneously would be an improvement. The system usability was highly appreciated by the users, with a global mean rating of 8.7 out of 10 . This score indicates that the tasks were performed satisfactorily. Some of the comments collect- 
ed to evaluate the graphic interface indicated that it could be improved in some ways. At the same time, participants suggested that the introduction of auditory information in the application menus would increase the usability and accessibility of the system.

- Service 5: Downloading of AD podcast.

The podcast service allows the users to download audio files and listen to them on a PC or a portable media device. These files only contain the AD audio channel of the selected programs. Once the files are downloaded, they can be copied to the portable audio player devices so that users can access the content wherever they want. The participants' task was to access the website, select and then play the podcasts. One of the goals of the evaluation was to find out whether there is a need for end-users to access podcasts of the programs they have previously missed.

Service usefulness was rated with a mean score of 8.3 out of 10 . In the questions regarding the positive aspects, users highlighted the time and location flexibility provided by podcasts. For example, one user said "if you are following a TV series, you can hear it in the car or while doing other things". Similarly, users stated that "podcasts might be very useful for blind people". The quality questionnaire comprised four Likert scale questions as well as one 5-alternative question regarding general quality. Quality was rated as "good" by most respondents, 64\%, and "excellent" by $29 \%$ of users. Similarly, in terms of their level of satisfaction, the system was rated very favorably by users, with a rating average of 8.4. Users evaluated all aspects concerning usability very positively. Almost all the questions were rated at 9 out of 10 or higher, indicating an excellent level of usability. The global average rating for usability was 9.1 points. These scores indicate that the system is easy to use when performing a podcast download. However, it is important to note that the question regarding the pleasantness of the graphic interface obtained a lower score (7.6 points), suggesting that the appearance of the display should be improved.

\section{CONCLUSIONS}

Five emerging services for AD were proposed and evaluated. We showed that these services are technically viable. They were tested through a user evaluation process. Users evaluated the usefulness, quality and usability of each service through questionnaires. Ratings and comments about the services were reported and analyzed. The project DTV4ALL has shown that it is technically feasible for IPTV and video on demand to incorporate $\mathrm{AD}$ emerging services. The usefulness of $\mathrm{AD}$ services has been validated by the users, not only as a tool to make content more accessible, but also as a means of reconciling the family. Evaluation showed that some attention should be paid to improving quality in order to enhance the experience of the user. Finally, usability has been clearly validated with respect to the ease of use and proposed improvements of the graphic interface.

We can conclude that the proposed services are widely accepted by users as an accessibility service, highly regarded as a social tool, and have the expected quality to 
encourage their deployment. The user evaluation process provided valuable information and feedback from participants. The results obtained at this stage will be compared with those obtained in the next evaluations, when more tests involving visually impaired users provide the necessary information to validate the current results.

\section{Acknowledgements.}

This research is supported by the grant from the Spanish Ministry of Science and Innovation FFI2009-08027, Subtitling for the Deaf and Hard of Hearing and Audio Description: objective tests and future plans; and also by the Catalan Government funds2009SGR700, and the EU project ADLAB.

\section{References.}

1. Kleinberger, T., Becker, M., Ras, E., Holzinger, A., Müller, P.: Ambient Intelligence in Assisted Living: Enable Elderly People to Handle Future Interfaces. In: Universal Access to Ambient Interaction. LNCS, vol. 4555, pp. 103-112. Springer, Heidelberg (2007)

2. Tanton, N.E. Ware, T. and Armstrong, M. (2006), 'Audio Description: what it is and how it works', BBC White paper, http://www.bbc.co.uk/rd/pubs/whp/whp051.shtml (accessed November 2011).

3. Oliveira, R., Ferraz de Abreu, J. and Almeida, A.M. (2011). 'An approach to identify requirements for an iTV audio description service'. In Proceedings of 9th European Conference EuroITV 2011 (Lisbon, June 2011), 227-230.

4. Braun, Sabine (2008): "Audiodescription Research: state of the art and beyond". Translation Studies in the New Millennium 6: 14-30.

5. Orero, Pilar (2007): "Sampling audio description in Europe". In Díaz Cintas, Jorge; Orero, Pilar \& Remael, Aline (eds.) Media for All: Subtitling for the Deaf, Audio Description and Sign Language. Amsterdam: Rodopi: 111-125.

6. Orero, Pilar (2005): “Audio Description: Professional Recognition, Practice and Standards in Spain”. Translation Watch Quarterly, Volume 1, Inaugural Issue, 7-18, December 2005, TSI: Melbourne.

7. Springett, M. and Griffiths, R. (2007). 'Accessibility of Interactive Television for Users with Low Vision - Learning from the Web'. In Proceedings of 5th European Conference EuroITV 2007 (Amsterdam, May 2007), 76-85.

8. Boyle, H., Nicolle, C., Maguire, M. and Mitchell, V. (2006) Older Users' Requirements for Interactive Television. in Clarkson, J., Langdon, P. and Robinson, P. eds. Designing Accessible Technology, Springer, 2006, pp: 85-92.

9. Digital Television For All (DTV4All). http://www.psp-dtv4all.org/

10. Lewis, James R. (1995) 'IBM computer usability satisfaction questionnaires: Psychometric evaluation and instructions for use', International Journal of Human-Computer Interaction, $7: 1,57-78$.

11. VLC media player (1.1.11). The VideoLan Project. http://www.videolan.org/ (accessed November 2011) 